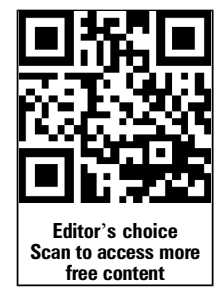

\title{
Microbial aetiology of healthcare associated pneumonia in Spain: a prospective, multicentre, case-control study
}

\author{
Eva Polverino, ${ }^{1,2}$ Antoni Torres, ${ }^{1,2}$ Rosario Menendez, ${ }^{2,3}$ Catia Cillóniz, ${ }^{1,2}$ \\ Jose Manuel Valles, ${ }^{4}$ Alberto Capelastegui, ${ }^{5} \mathrm{M}$ Angeles Marcos, ${ }^{6}$ \\ Inmaculada Alfageme, ${ }^{7}$ Rafael Zalacain, ${ }^{2,8}$ Jordi Almirall, ${ }^{2,9}$ Luis Molinos, $^{10}$ \\ Salvador Bello, ${ }^{2,11}$ Felipe Rodríguez, ${ }^{2,12}$ Josep Blanquer, ${ }^{2,13}$ Antonio Dorado, ${ }^{2,14}$ \\ Noelia Llevat, ${ }^{15}$ Jordi Rello, ${ }^{2,16}$ HCAP Study investigators
}

\begin{abstract}
- Additional material is published online only. To view please visit the journal online (http://dx.doi.org/10.1136/ thoraxjn-2013-203828).

For numbered affiliations see end of article.
\end{abstract}

\section{Correspondence to}

Dr Antoni Torres, Department of Pneumology, Hospital Clinic, Villarroel 170, Barcelona 8036, Spain; atorres@clinic.ub.es

Received 3 May 2013 Revised 9 August 2013 Accepted 28 August 2013

\section{Linked}

- http://dx.doi.org/10.1136/ thoraxjnl-2013-204060

To cite: Polverino $\mathrm{E}_{\text {, }}$

Torres A, Menendez $R$, et al.

Thorax 2013;68:

1007-1014.

\section{ABSTRACT}

Introduction Healthcare-associated pneumonia (HCAP) is actually considered a subgroup of hospital-acquired pneumonia due to the reported high risk of multidrug-resistant pathogens in the USA. Therefore, current American Thoracic Society/ Infectious Diseases Society of America guidelines suggest a nosocomial antibiotic treatment for HCAP. Unfortunately, the scientific evidence supporting this is contradictory.

Methods We conducted a prospective multicentre case-control study in Spain, comparing clinical presentation, outcomes and microbial aetiology of HCAP and community-acquired pneumonia (CAP) patients matched by age ( \pm 10 years), gender and period of admission ( \pm 10 weeks).

Results 476 patients (238 cases, 238 controls) were recruited for 2 years from June 2008. HCAP cases showed significantly more comorbidities (including dysphagia), higher frequency of previous antibiotic use in the preceding month, higher pneumonia severity score and worse clinical status (Charslon and Barthel scores). While microbial aetiology did not differ between the two groups (HCAP and CAP: Streptococcus pneumoniae: $51 \%$ vs $55 \%$; viruses: $22 \%$ vs $12 \%$; Legionella: $4 \%$ vs $9 \%$; Gram-negative bacilli: $5 \%$ vs $4 \%$; Pseudomonas aeruginosa: $4 \%$ vs $1 \%$ ), HCAP patients showed worse mortality rates (1-month: HCAP, 12\%; CAP 5\%; 1-year: HCAP, 24\%; CAP, 9\%), length of hospital stay (9 vs 7 days), 1 -month treatment failure ( $5.5 \%$ vs $1.5 \%)$ and readmission rate (18\% vs $11 \%)$ $(p<0.05$, each).

Conclusions Despite a similar clinical presentation, HCAP was more severe due to patients' conditions (comorbidities) and showed worse clinical outcomes. Microbial aetiology of HCAP did not differ from CAP indicating that it is not related to increased mortality and in Spain most HCAP patients do not need nosocomial antibiotic coverage.

\section{INTRODUCTION}

The current American Thoracic Society/Infectious Diseases Society of America (ATS/IDSA) guidelines for nosocomial pneumonia (2005) describe a new

\section{Key messages}

What is the key question?

- This multicentre case-control study investigates the microbial aetiology of HCAP showing that MDR infections are infrequent in Europe and that a nosocomial antibiotic coverage is not justified in these patients.

What is the bottom line?

- The poor outcomes observed in HCAP are clearly a consequence of the patients' poor clinical condition (comorbidities).

\section{Why read on?}

- There results possibly indicate the need for a closer medical follow-up rather than the use of broad-spectrum antibiotics.

clinical entity, healthcare-associated pneumonia (HCAP), which includes a number of heterogeneous conditions possibly associated with a high risk of multidrug-resistant (MDR) infections and mortality. ${ }^{1}$

This new clinical entity (HCAP) indirectly reflects substantial progress in healthcare (day hospital, outpatient clinics, etc), and profound demographic (life expectancy) and sociocultural changes in the general population. ${ }^{2-4}$ Indeed, the amount of long-term care facility patients has rapidly increased in recent years, pneumonia being one of the most frequent causes of hospitalisation and mortality. ${ }^{2-4}$ Different studies have shown that the incidence, clinical presentation and mortality for pneumonia in HCAP patients are similar to hospital-acquired pneumonia. ${ }^{1-5-7}$ Similar outcomes have been described for patients fulfilling other HCAP criteria such as patients receiving haemodialysis, previous antibiotic therapy, or immunocompromised patients. ${ }^{5-11}$ Unfortunately, there is no general consensus about the microbial aetiology of HCAP and its influence on outcomes. Whereas in the USA and Asia a high prevalence of MDR pneumonia (ie, Gram-negative bacilli or 
Staphylococcus aureus) has been described, in Europe the HCAP concept is progressively weakening due to limited evidence of 'nosocomial pathogens'. 12-22

Significant differences in local healthcare organisation and in population composition could be used to explain such microbiological disparities.

Finally, the two major concerns about HCAP are: (1) the excessive heterogeneity of HCAP definition (ie, immunosuppressed patients); and (2) the inadequacy of HCAP definition to predict the risk of MDR infections. ${ }^{23} 24$

For these reasons we performed a prospective case-control investigation on the microbial aetiology of HCAP in order to confirm the reliability of the HCAP concept in Europe and the role of its microbial aetiology in determining clinical outcomes.

\section{MATERIALS AND METHODS \\ Study subjects}

All consecutive immunocompetent adult patients hospitalised in the 12 collaborating Spanish hospitals with a suspicion of pneumonia were considered for recruitment between June 2008 and June 2010.

Two groups of patients were studied:

1. Patients with HCAP according to ATS/IDSA criteria. ${ }^{1}$ HCAP criteria for inclusion were slightly modified in order to focus the investigation on the risk of MDR pathogens in this population: the time of 'previous hospitalisation' was restricted to the last 2 months (instead of three); the following criteria were added: permanent nasogastric tube; gastrostomy; permanent catheters; permanent urinary catheter.

2. Selected 'control' community-acquired pneumonia (CAP) patients. Matching criteria were: age ( \pm 10 years), gender, and the hospitalisation period ( \pm 10 weeks) in order to respect patients' demographics and seasonal variations in microbial aetiology of pneumonia (flu, etc).

Exclusion criteria were: all causes of immunosuppression (HIV, neoplasia, etc, excluding steroids), no written consent for study recruitment.

Witnessed bronchoaspiration pneumonia (the inhalation of oropharyngeal or gastric contents into the lower respiratory tract) was not considered for the study because chemical pneumonitis could not be ruled out.

\section{Data collection}

Data were collected on age, gender, smoking and alcohol habits $(80 \mathrm{~g} /$ day, corresponding to approximately 11 of wine, eight standard-sized beers, or one-half pint of hard liquor each day), previous influenza and pneumococcal vaccinations, chronic comorbidities (cardiovascular, hepatic, renal, rheumatological, dementia and neurological diseases, diabetes mellitus, neoplasia in remission, etc), modified Charlson index and Barthel scale (see tables E1 andE2 in supplementary data, available online only) to assess comorbidities and physical activity, respectively, chronic therapy (inhaled and systemic corticosteroids, gastroprotective drugs and statins), previous pneumonia, previous antibiotic therapy in the last month and aspiration evidence (dysphagia scale, see table E3 in supplementary data, available online only).

Main clinical signs and symptoms (cough, expectoration, etc) were recorded at admission, as well as analytical data (leucocyte count, C- reactive protein, etc), pneumonia severity index (PSI) and CURB-65 scores (confusion, blood urea, respiratory rate, blood pressure, age $>65$ years), radiological data (multilobar infiltration, etc), antimicrobial treatment (with reference to Spanish CAP guidelines) and use of systemic corticosteroids. ${ }^{25-27}$
Pulmonary (pleural effusion, empyema, atelectasis, cavitations, respiratory distress, pneumothorax and surgical pleural draining) and extrapulmonary complications of pneumonia (septic shock, acute renal failure, endocarditis, meningitis, cardiac arrhythmias, diarrhoea, myocardial infarction, cardiac arrest, pancreatitis) were also recorded, as well as 30-day, 90-day and 1-year mortality rate (and cause of death), hospital length of stay (LOS), clinical stability, intensive care unit (ICU) admission and ventilatory support (invasive or non-invasive mechanical ventilation, MV) requirement, treatment failure and 30-day and 1-year hospital readmissions. ${ }^{28}$

\section{Patient follow-up}

Surviving patients underwent a radiological and serological follow-up at 4-6 weeks. Serial phone calls were performed every 3 months up to 1 year after admission in order to assess eventual readmissions, new antibiotic treatments, pneumonia complications or fatality cases and their causes.

\section{Microbiological investigations}

Routine sampling to determine the microbial aetiology of pneumonia included sputum, urine for Streptococcus pneumoniae and Legionella pneumophila antigens, blood cultures, nasopharyngeal swabs for respiratory viruses and for methicillin-resistant $S$ aureus (MRSA). Pleural puncture, tracheobronchial aspirates (TBAS) and bronchoalveolar lavage, when available, were collected for Gram and Ziehl-Nielsen stains and for cultures for bacterial, fungal and mycobacterial pathogens. We only considered valid sputum samples according to current microbiological standards. ${ }^{29}$ Positive swabs for MRSA were not considered indicative of MRSA-induced pneumonia if this potential aetiology was not confirmed by a positive culture of any other respiratory sample (sputum, TBAS, etc). Serology tests for atypical pathogens were performed at admission and after 4-6 weeks (see supplementary data, available online only).

In case of failure to respond, a new microbiological investigation was conducted for bacterial, fungal and mycobacterial pathogens.

\section{Sample processing}

Sample processing techniques have been described previously. ${ }^{30}$ The following respiratory viruses were studied through a reverse transcriptase PCR technique (CLART-PneumoVir, Genomica; see supplementary data, available online only): adenovirus; bocavirus; coronavirus; enterovirus (echovirus); influenza virus A (subtypes H3N2 human, H1N1 human, B, C and H1N1/2009); metapneumovirus (subtypes A and B); parainfluenza virus 1, 2, 3 , and 4 (subtypes $A$ and $B$ ); rhinovirus; respiratory syncytial virus type A (VSR-A) and B (VSR-B).

\section{Statistical analysis}

A descriptive analysis of all variables was performed, analysing qualitative variables by means of absolute and relative frequencies, while quantitative variables have been represented by means, SD and CI if normally distributed, or through medians, minimal and maximal values and IQR if they did not follow a Gaussian distribution.

Student's t test (parametric) and the Mann-Whitney (nonparametric) test were used to assess significant differences in quantitative variables. For qualitative variables, Pearson's $\chi^{2}$ test, or Fisher exact test for tables $(2 \times 2)$ and tests of likelihood ratios for tables $(\mathrm{m} \times \mathrm{n})$ were used. ${ }^{31}$ Student's $\mathrm{t}$ and Wilcoxon tests were used for associations of paired data in quantitative variables. For qualitative variables $\mathrm{McNemar}$ tests for tables 
$(2 \times 2)$, or signs tests for tables $(m \times n)$ were used. A multivariate logistic regression analysis was performed to identify factors associated with short-term and long-term mortality. First, univariate relationships were analysed, in order to identify the possible factors associated with the event (death). Second, different models were developed with significant variables. Several sets of significant variables were introduced into the model as it was not possible to use them all at once, given the large sample size, and no confounder variables were found. After adjusting several models, one of the models with best adjustment was chosen. 95\% CI for estimates and SPSS V.17.0 statistical pack were used.

\section{RESULTS}

\section{Patients' characteristics}

We recruited 476 patients (238 HCAP cases and 238 CAP controls). The main HCAP patients fulfilled one HCAP criterion $(n=207,87 \%)$ while $9.7 \%(n=23)$ met two criteria, 3\% $(n=8)$ three or more criteria.

The most frequent inclusion criterion was residency in longterm care facilities $(n=119 ; 50 \%)$, followed by recent hospitalisation $(n=100 ; 42 \%)$, domiciliary wound care $(n=22 ; 9.2 \%)$, haemodialysis $(n=11 ; 4.6 \%)$, permanent urinary tube $(n=10$; $4.2 \%)$, gastrostomy $(n=9 ; 3.8 \%)$, nasogastric tube $(n=4 ; 1.7 \%)$, permanent catheter $(n=2 ; 0.8 \%)$ and close contact with MDR infection $(n=1 ; 0.4 \%)$.

Most patients were hospitalised in pulmonology departments (HCAP: 77.3\% CAP: 84.5\%), while fewer patients were hospitalised in internal medicine departments (HCAP: 17.6\%; CAP: 11.8\%) or other services (HCAP: $8.9 \%$; CAP: 6.3\%).

The main patients' characteristics are shown in table 1 . The two groups did not differ in vaccination rates (flu and pneumococcal), smoking and alcohol history, chronic use of steroids (both inhaled and systemic) or in the percentage of previous pneumonia. By contrast, HCAP patients showed a more frequent use of pump inhibitors and of antibiotics in the last month before pneumonia (table 1). The most frequent antibiotics previously administered were: fluoroquinolones in both groups (HCAP: 29 (44\%) vs CAP: 11 (41\%); NS), $\beta$-lactams (HCAP: $20(30.3 \%)$ vs CAP: 8 (29.6\%); NS) and macrolides (HCAP: 1 (1.5\%) vs CAP: 3 (11.1\%); NS).

Table 1 Patients' main characteristics

\begin{tabular}{|c|c|c|c|c|c|}
\hline $\mathbf{N}$ & \multicolumn{2}{|c|}{ Cases (HCAP) } & \multicolumn{2}{|c|}{$\begin{array}{l}\text { Controls } \\
\text { (CAP) }\end{array}$} & p Value \\
\hline Men, \% & \multicolumn{2}{|l|}{68} & \multicolumn{2}{|l|}{68} & \\
\hline Age, mean $\pm S D$ (years) & \multicolumn{2}{|c|}{$78.8 \pm 10.8$} & \multicolumn{2}{|c|}{$77.7 \pm 10.1$} & NS \\
\hline Current smokers, n (\%) & 21 & $8.8 \%$ & 25 & $10.5 \%$ & NS \\
\hline Ex-smokers, n (\%) & 102 & $42.9 \%$ & 100 & $42.0 \%$ & \\
\hline Alcohol intake $>80$ g/day, n (\%) & 3 & $1 \%$ & 7 & $3 \%$ & NS \\
\hline Flu vaccine, $\mathrm{n}(\%)$ & 143 & $63 \%$ & 152 & $68 \%$ & NS \\
\hline Pneumococcal vaccine, $\mathrm{n}(\%)$ & 41 & $18 \%$ & 30 & $13 \%$ & NS \\
\hline Previous pneumonia, n (\%) & 73 & $32 \%$ & 68 & $29 \%$ & NS \\
\hline $\begin{array}{l}\text { Previous antibiotic therapy } \\
\text { (last month) }\end{array}$ & 78 & $33 \%$ & 34 & $15 \%$ & 0.000 \\
\hline Inhaled cortisteroids, n (\%) & 51 & $21 \%$ & 48 & $20 \%$ & NS \\
\hline Systemic steroids, n (\%) & 21 & $9 \%$ & 18 & $8 \%$ & NS \\
\hline Statins & 52 & $22 \%$ & 49 & $21 \%$ & NS \\
\hline Proton-pump inhibitors, n (\%) & 124 & $52 \%$ & 105 & $44 \%$ & 0.048 \\
\hline Anti- $\mathrm{H}_{2}$ drugs & 25 & $17 \%$ & 8 & $7.1 \%$ & 0.017 \\
\hline
\end{tabular}

The median number of comorbidities was clearly higher in HCAP due to the higher rate of neurological disorders in contrast with other diseases (respiratory, cardiac, diabetes, etc) that showed similar distribution between groups (table 2). Concordantly, in HCAP dysphagia was more frequent while body mass index decreased in comparison with CAP.

Among the respiratory diseases, chronic obstructive pulmonary disease (COPD) was the most frequent in both groups, followed by bronchiectasis and asthma. As a consequence, patients with a high severity score (PSI classes IV-V 85\% in HCAP vs $68 \%$ in CAP; $p=0.000$ ) were more frequent in the HCAP group than in CAP indicating an increased mortality risk (see figure E1 in supplementary data, available online only).

HCAP patients had a higher mean Charlson score for comorbidities (cases: 2.47, 95\% CI 2.25 to 2.69 vs controls: $1.67,95 \%$ CI 1.47 to $1.86 ; p=0.000)$ and lower Barthel index for autonomy (cases: 57.9 points, $95 \%$ CI 53.1 to 62.8 vs controls: $83.7,95 \%$ CI 80.2 to $87.3 ; \mathrm{p}=0.000$ ) than CAP (figure 1 ), indicating a poorer performance status.

Signs (radiological extension, oxygenation, etc) and symptoms (fever, dyspnoea, etc) of presentation were similar for both groups, with the only exception of hypothermia, altered mental status (both more frequent among HCAP) and chills (less frequent in HCAP) (table 3).

Despite higher initial PSI, HCAP patients showed similar rates of pulmonary complications (pleural effusion, etc), ICU admission and MV (cases, 3\% vs controls, $2 \% ; \mathrm{p}=\mathrm{NS}$ ) but a trend towards more extrapulmonary complications (septic shock, etc) globally than CAP (table 3 ).

The HCAP subgroups of 'nursing home' (NH) and of 'recent hospitalization' (RH) showed a trend towards an increased incidence of respiratory diseases (COPD, NH: 67\%; RH: 57\%; HCAP: $30 \%)$ and of both inhaled (NH: 68\%; RH: 67\%; HCAP: 21\%) and systemic steroids (NH: 32\%; RH: 33\%; HCAP: 9\%).

\section{Microbiological findings}

Most patients underwent a broad microbiological investigation (see table E4 in supplementary data, available online only), with at least one positive microbiological result in 83 cases $(34.9 \%)$ and 72 controls $(30.3 \%)$ but two or three different

Table 2 Patients' characteristics at presentation

\begin{tabular}{lccccl}
\hline & \multicolumn{2}{c}{ Cases (HCAP) } & Controls (CAP) & p Value \\
\hline BMI, media \pm SD & 25.6 & 4.3 & 26.8 & 4.8 & 0.029 \\
Comorbidities $\geq 2, \mathrm{n}(\%)$ & 158 & $75 \%$ & 102 & $53 \%$ & 0.000 \\
$\quad$ Respiratory disease, n (\%) & 118 & $50 \%$ & 111 & $48 \%$ & NS \\
COPD & 72 & $30 \%$ & 61 & $26 \%$ & NS \\
Bronchiectasis & 12 & $5 \%$ & 6 & $2.5 \%$ & NS \\
Asthma & 9 & $4 \%$ & 3 & $1.2 \%$ & NS \\
Diabetes mellitus & 57 & $27 \%$ & 55 & $28 \%$ & NS \\
Cardiac failure & 94 & $45 \%$ & 99 & $51 \%$ & NS \\
Neurological diseases, n (\%) & 114 & $54 \%$ & 46 & $24 \%$ & 0.000 \\
Dysphagia (1-4), n (\%) & 90 & $39 \%$ & 33 & $14 \%$ & 0.000 \\
PSI, mean (SD) & 123 & 33 & 104 & 29 & 0.000 \\
PSI I-III, n (\%) & 35 & $15 \%$ & 75 & $33 \%$ & 0.000 \\
PSI IV-V, n (\%) & 198 & $85 \%$ & 156 & $68 \%$ &
\end{tabular}

BMI, body mass index; CAP, community-acquired pneumonia; COPD, chronic obstructive pulmonary disease; HCAP, healthcare-associated pneumonia; PSI, pneumonia severity index. 
Figure 1 Patients' distribution according to modified Charlson and Barthel indexes.
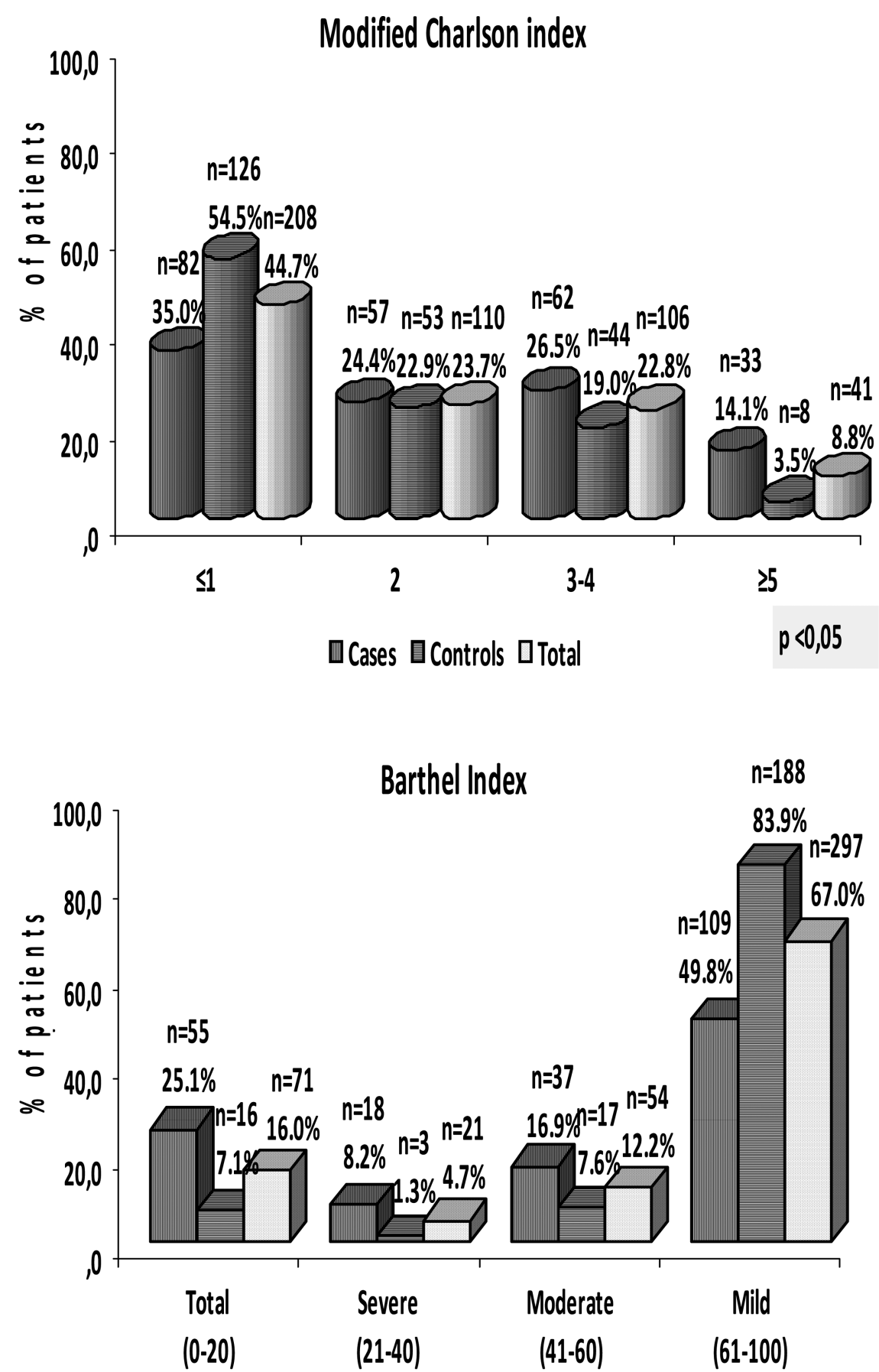

$\square$ Cases 貝Controls QTotal microorganisms were found contemporarily in 11 cases $(10.7 \%)$ and in 10 controls $(10.9 \%)(\mathrm{p}=0.200)$.

$S$ pneumoniae was the most frequent isolated pathogen $(63 \%$ vs $71 \%)$ followed by respiratory viruses $(27 \%$ vs $15 \%)$, $L$ pneumophila (5\% vs 11\%) Gram-negative bacilli (including Escherichia coli, Klebsiella pneumoniae, Acinetobacter lwoffi, Proteus mirabilis) (7\% vs 6\%), P aeruginosa (5\% vs $1 \%$ ), methicillin-sensitive $S$ aureus (2\% vs $3 \%$ ) and atypical pathogens (including Mycoplasma pneumoniae, Coxiella burnetii and Chlamydia pneumoniae) (2\% vs $4 \%$ ). All isolated pathogens were similarly distributed between cases and controls but respiratory viruses showed a trend towards increased frequency among cases while L pneumophila and other atypical pathogens showed a trend to be more frequent among controls. We did not observe any specific predominance among the different respiratory viruses identified in both groups (see table E5 in supplementary data, available online only). Among potential MDR pathogens $P$ aeruginosa showed only a mild non-significant trend towards a higher prevalence among cases with Gram-negative bacilli (table 4). The other Gram-negative isolates corresponded to five cases (one extended-spectrum betalactamase (ESBL) resistant $E$ coli, two non-MDR $E$ coli, one non-MDR A lwoffii, one non-MDR $P$ mirabilis) and four controls (one ESBL-resistant E coli, three non-MDR E coli). 
Table 3 Clinical presentation of HCAP and CAP patients

\begin{tabular}{|c|c|c|c|c|c|}
\hline \multirow[b]{2}{*}{ Fever, n (\%) } & \multicolumn{2}{|c|}{$\begin{array}{l}\text { Cases } \\
\text { (HCAP) }\end{array}$} & \multicolumn{2}{|c|}{$\begin{array}{l}\text { Controls } \\
\text { (CAP) }\end{array}$} & \multirow{2}{*}{$\begin{array}{l}\text { p Value } \\
\text { NS }\end{array}$} \\
\hline & 161 & 68 & 168 & 71 & \\
\hline Hypothermia, n (\%) & 16 & 7 & 4 & 2 & 0.005 \\
\hline Chills, n (\%) & 66 & 28 & 92 & 39 & 0.016 \\
\hline Cough, n (\%) & 161 & 68 & 176 & 74 & NS \\
\hline Purulent phlegm, n (\%) & 67 & 51 & 73 & 51 & NS \\
\hline Digestive symptoms, $\mathrm{n}(\%)$ & 38 & 16 & 25 & 11 & NS \\
\hline Dyspnoea, n (\%) & 168 & 71 & 155 & 65 & NS \\
\hline Altered mental status, $\mathrm{n}(\%)$ & 63 & 27 & 34 & 14 & 0.001 \\
\hline $\mathrm{PaO}_{2}<65 \mathrm{~mm} \mathrm{Hg}$ & 146 & 72 & 122 & 66 & NS \\
\hline Bacteraemia & 24 & 10.1 & 19 & 8.0 & NS \\
\hline Multilobar infiltration, $\mathrm{n}(\%)$ & 57 & 24 & 47 & 20 & NS \\
\hline ICU admission, n (\%) & 10 & 4 & 11 & 5 & NS \\
\hline NIV, n (\%) & 10 & 4 & 12 & 5 & NS \\
\hline$M V, n(\%)$ & 7 & 3 & 5 & 2 & NS \\
\hline Respiratory complications & 37 & 15.5 & 38 & 16.0 & NS \\
\hline Distress & 7 & 2.9 & 7 & 18.4 & NS \\
\hline Empyema & 2 & 0.8 & 4 & 10.5 & NS \\
\hline Pleural effusion, $\mathrm{n}(\%)$ & 26 & 10.9 & 26 & 68.4 & NS \\
\hline Cavitation & 2 & 0.8 & 1 & 2.6 & NS \\
\hline Non-respiratory complications & 92 & 38.6 & 62 & 26 & 0.005 \\
\hline Renal failure & 33 & 13.8 & 20 & 8.4 & NS \\
\hline Septic shock & 11 & 4.6 & 6 & 2.5 & NS \\
\hline Arrhythmia & 11 & 4.6 & 7 & 2.9 & 1.00 \\
\hline Diarrhoea & 9 & 3.8 & 6 & 2.5 & 0.785 \\
\hline Others & 28 & 11.8 & 23 & 9.7 & 0.72 \\
\hline
\end{tabular}

CAP, community-acquired pneumonia; HCAP, healthcare-associated pneumonia; ICU, intensive care unit; MV, mechanical ventilation; NIV, non-invasive ventilation; $\mathrm{PaO}_{2}$, arterial oxygen tension.

The nasopharyngeal swab identified 16 cases of MRSA (13\% of all isolates) among HCAP and seven among CAP (6\%). A mixed infection was detected in $11 \%$ of the total population

Table 4 Microbiological aetiology of pneumonia among patients with known aetiology

\begin{tabular}{|c|c|c|c|c|c|}
\hline & \multicolumn{2}{|c|}{$\begin{array}{l}\text { Cases } \\
\text { (HCAP) }\end{array}$} & \multicolumn{2}{|c|}{$\begin{array}{l}\text { Controls } \\
\text { (CAP) }\end{array}$} & \multirow[b]{2}{*}{$\mathrm{p}$ Value } \\
\hline & $\mathrm{N}$ & $\%$ & $\mathrm{~N}$ & $\%$ & \\
\hline Streptococcus pneumoniae & 52 & 62.7 & 51 & 70.8 & 0.282 \\
\hline Respiratory Viruses & 22 & 26.5 & 11 & 15.3 & 0.116 \\
\hline Legionella pneumophila & 4 & 4.8 & 8 & 11.1 & 0.227 \\
\hline Gram-negative bacilli* & 5 & 7.2 & 4 & 5.6 & 1.000 \\
\hline Pseudomonas aeruginosa & 4 & 4.8 & 1 & 1.4 & 0.373 \\
\hline MSSA & 2 & 2.4 & 2 & 2.8 & 1.000 \\
\hline Atypical pathogens & 2 & 2.4 & 3 & 4.2 & 0.664 \\
\hline Haemophilus influenza & 1 & 1.2 & 1 & 1.4 & 1.000 \\
\hline Moraxella catarrhalis & 1 & 1.2 & 0 & .0 & 1.000 \\
\hline Total number & 83 & & 72 & & \\
\hline
\end{tabular}

${ }^{*}$ Gram-negative bacilli include: Escherichia coli, Klebsiella pneumoniae, Acinetobacter Iwoffii, Proteus mirabilis. Only two of them were multidrug resistant (one case and one control of ESBL-resistant $E$ coli).

†Methicillin-sensitive Staphylococcus aureus.

CAP, community-acquired pneumonia; ESBL, extended-spectrum beta-lactamase HCAP, healthcare-associated pneumonia; Respiratory viruses include: respiratory syncytial virus, rhinovirus, adenovirus, influenza virus $A$ and $B$, parainfluenza virus IIV, metapneumovirus A and B, enterovirus; Atypical pathogens include: Mycoplasma pneumoniae, Chlamydia pneumophila, Coxiella burnetii. $(\mathrm{n}=21)$, corresponding to 11 cases $(11 \%$ of cases with aetiology) and 10 controls (11\% of controls) with a combination of two different micro-organisms (five cases and five controls with two different bacteria, two cases and four controls with a bacteria plus a virus, one case and one control with two viruses) and only three cases of three different pathogens (a bacteria plus two viruses in two cases, and three bacteria in one case) (see table E6 in supplementary data, available online only).

Most patients $(n=424 ; 92 \%)$ received an antibiotic coverage consistent with Spanish CAP guidelines, with a mild not significant trend towards greater adherence for CAP (antibiotic adequacy: HCAP, $89.6 \%$; CAP 94.3\%; $p=0.085$ ). Only $11 \%$ of the cases of pneumonia who did not receive recommended antibiotic therapy were caused by specific antibiotic allergies, while $5 \%$ of the cases were modified according to microbiological results.

\section{Clinical course and outcomes}

The clinical outcomes of HCAP were clearly poorer than for CAP as both mean LOS $(9 \pm$ SD 8 days vs $7 \pm 6$ days, $p=0.008)$ and time to clinical stability $(5.7 \pm$ SD 4.6 days vs $4.8 \pm 3.6$ days, $\mathrm{p}=0.030$ ) were worse for HCAP, as were the rates of 30-day treatment failure (HCAP, 11cases $(5.5 \%)$ vs CAP, three cases $(1.5 \%) ; \mathrm{p}=0.031$ ) and of 30-day readmissions (HCAP, $\mathrm{n}=37$ $(18 \%)$ vs CAP, $\mathrm{n}=22(11 \%) ; \mathrm{p}=0.030)$, the majority being for a respiratory cause $(51 \%$ among $\mathrm{HCAP}, 55 \%$ among CAP). The rate of readmissions remained higher for HCAP than for CAP during the entire study follow-up period (3 months, $22 \%$ vs $14 \%, \mathrm{p}=0.054 ; 12$ months, $43 \%$ vs $21 \%, \mathrm{p}=0.000)$, with a clear prevalence of respiratory causes $(59-65 \%)$.

The mortality rate was also increased for HCAP patients at both 1-month (HCAP, $\mathrm{n}=29$ (12.2\%) vs CAP, $\mathrm{n}=11 \quad(4.6 \%)$; $\mathrm{p}=0.005)$ and 12-month follow-up (HCAP, $\mathrm{n}=58$ (24.4\%) vs CAP, $\mathrm{n}=22(9.2 \%) ; \mathrm{p}<0.001)$.

Among patients who died during the study four cases (14\%) and three controls (27\%) were admitted to ICU, while six cases received MV (21\%; three non-invasive MV, three invasive MV) and six controls (55\%; four non-invasive MV, two invasive MV).

We analysed mortality rates across aetiological subgroups and we could only find higher 1-year mortality for $S$ pneumoniae infection (HCAP, $10(19.2 \%)$ vs CAP, one $(2 \%) ; \mathrm{p}=0.008)$ and a slight non-significant trend towards increased 1-year mortality for respiratory viruses (HCAP, six (27.3\%) vs CAP, two (18.2\%), $\mathrm{p}=0.68$ ) among HCAP. Other aetiologies showed only very few fatality cases and did not show any statistical differences.

While LOS, readmission and 1-month mortality rates from $\mathrm{NH}$ and $\mathrm{RH}$ subgroups were similar to the whole HCAP population, treatment failure (12.9\% vs $5.5 \%)$ and the 1 -year mortality rate $(27 \%$ vs $24 \%)$ showed an increasing trend in the $\mathrm{RH}$ group.

\section{Predictors of mortality}

The multivariate analysis identified the following risk factors for 30-day mortality: a chronic cardiac disease (OR 9.95, 95\% CI 2.84 to 34.94; $\mathrm{p}=<0.01$ ), an increased LOS (OR 1.10) and a decreased autonomy at Barthel score (OR 0.97), a respiratory rate greater than 30 breaths per min (OR 1.16). Only the presence of an 'alveolar' pulmonary infiltrate at chest X-ray was a protective factor in comparison with a different pattern (interstitial or mixed pattern) (OR 0.16) (see table E7 in supplementary data, available online only).

The risk factors associated with 1-year mortality were: HCAP (OR 3.15), increasing age (+5 years, OR 1.29), prolonged ICU stay (+1 day, OR 1.26), increased respiration rate ( $>30$ per 
min; OR 2.16). Only the presence of pleuritic pain was a protective factor (OR 0.40) (see table E8 in supplementary data, available online only).

We also adjusted the mortality rates for multivariate analysis for all relevant comorbidities (heart failure, neurological, diabetes, chronic respiratory disease, liver and renal diseases) to assess their role but no one of them individually achieved the statistical significance to enter the multivariate analysis. Nevertheless, the HCAP definition was associated with a twofold increased risk of mortality (OR 2.2, 95\% CI 1.21 to 4.07; $\mathrm{p}=0.010)$. On the other hand, the Barthel index for autonomy was a protective factor for mortality (OR 0.99, 95\% CI 0.98 to $0.99 ; \mathrm{p}<0.01)$.

\section{DISCUSSION}

The main findings of this study are:

1. HCAP patients showed worse functional status than CAP patients despite similar age and clinical presentation.

2. Concordantly to initial PSI, HCAP clinical outcomes were clearly worse than for CAP; a low Barthel score was associated with increased 1-month mortality.

3. The microbiological investigation showed similar patterns in both groups indicating that microbial aetiology is not the leading cause of increased mortality from HCAP in Spain.

HCAP patients clearly differ from CAP patients despite similar age and gender due to the poorer functional status, as reflected by the number of comorbidities and level of autonomy for daily activities. In particular, HCAP patients showed a higher frequency of neurological disorders and dysphagia (although no anaerobic pathogens were found), particularly among NH patients. Moreover, a higher proportion of HCAP patients had recently received antibiotics before an episode of pneumonia and chronically use gastric acidity inhibitors, a recognised risk factor for pneumonia. ${ }^{32-34} \mathrm{NH}$ and $\mathrm{RH}$ subgroups showed an increased frequency of COPD and, in parallel, of steroid use (both systemic and inhaled), a recognised risk factor for pneumonia.

The clinical presentation was very similar between the two study groups with the only exception being hypothermia, which was more frequent among HCAP, and chills, which was more frequent among CAP, indicating globally that a clinical distinction between the two groups is almost impossible at medical examination. Nevertheless, the PSI score was in general higher for HCAP patients, reflecting the relevant weight of comorbidities on initial pneumonia severity. Interestingly, the rate of septic shock of HCAP patients was slightly higher than the rate of ICU suggesting indirectly some treatment restriction for these patients. In general, a trend towards limited ICU admission and MV use was observed among HCAP patients, possibly reflecting a tendency towards a limited use of life-supporting measures or poorer patient conditions.

The main clinical outcomes clearly reflected the PSI estimation because HCAP showed increased mortality in both the short and long term, such as worse LOS, readmissions, etc.

The multivariate analyses showed that HCAP is more clearly associated with long-term mortality (1 year) rather than with short term (1 month), suggesting a predominant role of poor functional status on main outcomes. As a confirmation, the Barthel score for functional status is more strictly correlated with mortality (multivariate analysis) than comorbidities globally (Charlson) and individually, suggesting that a low Barthel score should indicate a more careful patient follow-up after discharge. Indeed, different authors have already described the prognostic relevance of a functional status assessment for mortality in CAP patients and, possibly, a simple assessment for mobility autonomy could help in follow-up decisions. ${ }^{35} 36$

Finally, the microbiological investigation demonstrated that despite the increased severity at admission and the higher use of antibiotics before the pneumonia episode, a known risk factor for MDR infection, HCAP patients showed an aetiological pattern not far from the typical CAP microbiology.

Nevertheless, a few interesting findings were observed: the role of viral infections is relevant among HCAP and is probably also related to bacterial entrance; ${ }^{37}$ nonetheless, it is worth remembering that the PCR technique detects genetic material instead of live microbes; the rates of Gram-negative bacilli and $P$ aeruginosa infections were similar between HCAP and CAP, discrediting the hypothesis of a 'nosocomial microbiological' pattern for HCAP in Spain and justifying the general Spanish trend to follow CAP guidelines for antibiotic treatment. Indeed, the choice of modified criteria for HCAP definition for this study was taken to ensure a wider selection of potential MDR risky conditions (by reducing the time since previous hospitalisation to 2 months and by adding other potential sources of MDR infections currently and surprisingly not considered by guidelines). Nevertheless, few cases of MDR infections were detected among both HCAP and CAP groups, suggesting that specific risk factors for MDR infections should be systematically investigated in the future to improve the antibiotic coverage and possibly reduce the related excess mortality. Shorr et al ${ }^{24}$ described an association between potential MDR pathogens and specific risk factors (ICU, etc). Indeed, in our series the few HCAP and CAP cases of potential MDR pathogens made this correlation with MDR risk factors not evaluable. ${ }^{24}$ A lower frequency of $L$ pneumophila was observed among HCAP possibly due to the limited mobility and reduced contagious risk of these patients.

Another important aspect of the study is the relatively high proportion of oral colonisation by MRSA, especially among HCAP (HCAP 16\%, CAP 6\%), that in association with the increased risk of broncho-aspiration, could create the alert of MRSA pneumonia in already compromised patients. Unfortunately, due to the bad clinical conditions of the main patients it was possible to perform only a minimal number of TBAS and bronchoalveolar lavage that would have considerably increased the rate of microbiological diagnosis and investigated the role of oral 'colonisers' in causing pneumonia through the aspiration of oropharyngeal secretions. Moreover, the percentage of microbiological findings is relatively low despite the investigators' efforts, due to the limited ability of many patients to expectorate.

By contrast, the main strengths of the study are: this is the first prospective multicentre case-control study conducted in Europe on an HCAP population; a wide microbiological investigation was systematically performed in all centres using identical methods; we systematically recorded patients' functional status and the long-term clinical outcomes (1 year).

We can therefore conclude that HCAP, according to the ATS/ IDSA definition, clearly corresponds to patients with poor functional status and with multiple comorbidities, who despite having a similar clinical presentation and similar microbial aetiology show worse clinical outcomes in both the short and long term. These findings perfectly match with other recent European studies (UK, Germany, Spain) and corroborate the idea that the usual CAP antibiotic coverage is still indicated in these patients in Europe. ${ }^{19} 2238$ Moreover, patients with HCAP criteria possibly deserve more attention during hospitalisation 
regarding poor functional status and comorbidities that could easily decompensate as a consequence of a respiratory infection; a more prolonged HCAP follow-up after hospital discharge is needed due to the higher long-term mortality risk.

However, all our findings do not support the use of HCAP definition in Spain because the clinical approach to very elderly or multimorbid patients (follow-up) should not depend on this classification and the antibiotic choice can still follow CAP guidelines. Risk factors for potential MDR infections should always be investigated.

\author{
Author affiliations \\ ${ }^{1}$ Servicio de Pneumologia, Hospital Clínic i Provincial de Barcelona, IDIBAPS, \\ Barcelona, Spain \\ ${ }^{2}$ CIBER de Enfermedades Respiratorias (Ciberes), Barcelona, Spain \\ ${ }^{3}$ Servicio De Neumología, Hospital La Fe, Valencia, Spain \\ ${ }^{4}$ Servicio de Urgencias, Hospital La Fe, Valencia, Spain \\ ${ }^{5}$ Servicio de Neumología, Hospital de Galdakao, Bilbao, Spain \\ ${ }^{6}$ Servicio de Microbiologia, Hospital Clínic i Provincial de Barcelona; Barcelona \\ Centre for International Health Research (CRESIB, Hospital Clinic-Universitat de \\ Barcelona), Barcelona, Spain \\ ${ }^{7}$ Servicio de Neumología, Hospital de Valme, Sevilla, Spain \\ ${ }^{8}$ Servicio de Neumología, Hospital Cruces, Bilbao, Spain \\ ${ }^{9}$ Cures Intensives, Consorcio Sanitario del Maresme, Mataró, Spain \\ ${ }^{10}$ Servicio de Neumología, Hospital Central de Astúrias, Spain \\ ${ }^{11}$ Servicio de Neumología, Hospital Miguel Servet, Zaragoza, Spain \\ ${ }^{12}$ Servicio de Neumología, Hospital Dr Negrin, Gran Canaria, Spain \\ ${ }^{13}$ Intensive Care Unit, Hospital Clínico Universitario de Valencia, Godella (Valencia), \\ Spain \\ ${ }^{14}$ Servicio de Neumología, Hospital Carlos Haya de Malaga, Málaga, Spain \\ ${ }^{15}$ Pfizer Spain, Madrid, Spain \\ ${ }^{16}$ Hospital Joan XXII of Tarragona and Hospital Vall Hebron (Critical Care \\ Department), Universitat Autonoma de Barcelona, Barcelona, Spain
}

Acknowledgements This multicentre study was made possible due to the effort of 12 different research Spanish groups whose collaborators all contributed to achieve the data shown in this manuscript.

Collaborators List of investigators: Hospital Clinic of Barcelona: Albert Gabarrus (statistician), Rebeca Domingo and Encarnación Moreno (research nurses). Hospita La Fe, Valencia: Dr Soledad Reyes. Hospital Joan XXII de Tarragona: Dr Emili Diaz (Critical Care Centre Corporació Sanitària I Universitaria Parc Taulí-Hospital De Sabadell), Frederic Gomez, PhD, Ana Parra, Elisabet Papiol, Dr Thiago Lisboa, MD. Hospital Central de Astúrias: Dr Ana Pando. Hospital Cruces, Bilbao: Dr Ainhoa Gomez. Hospital Dr Negrin, Gran Canaria: Dr Annety Juanes Olite. Hospital Clínico Universitario de Valencia: Dr Marisa Briones, Dr Magnolia Nieto. Hospital Miguel Servet, Zaragoza: Dr Vera Solsona, Dr Minchole Lapuente. Hospital Carlos Haya de Malaga: Dr Juan Carlos Martin. Hospital de Valme, Sevilla: Dr Javier Gallego. Hospital Galdakao-Usansolo: Dr Pedro P. España, Dr Inmaculada Gorordo and Dr Ane Uranga. Consorcio Sanitario del Maresme, Mataró: Dr Mari de La Torres, Dr Goretti Sauca, Ivana Anglada (research nurses).

Contributors EP is the coordinator and main investigator of the study, directly involved in the project design, patient recruitment, data collection and analysis, and is the main author of the manuscript. The following authors were main investigators in the relative collaborating centres and directly involved in patient recruitment and data collection and reviewed and approved the final version of the manuscript: $\mathrm{CC}$, $J M V, A C, I A, R Z, J A, L M, S B, F R, J R, J B$ and $A D$. MAM contributed to the project design, supervised the microbiological data of the study, and approved the final version of the study. RM contributed to the design of the project, and contributed to and approved the final manuscript. AT led the study group, contributed to the design of the project, and contributed to and approved the final study; he is the guarantor of the entire study.

Funding This study was sponsored by Pfizer. FIS PI080240, Pfizer, Genomica, Brahms, SEPAR2008, SOCAP2008, Ciberes (CB06/06/0028), Ciberes es una iniciativa del ISCIII, 2009SGR911, IDIBAPS, FIS PI080448, PI08/0123, FIS PI0804520, Proyecto Integrado de Investigación (PII) Infecciones Respiratorias de la vías bajas de la SEPAR 2005-2010.

Competing interests None.

Patient consent Obtained.

Ethics approval Ethics approval was received from the ethics committees of all the local hospitals.

Provenance and peer review Not commissioned; externally peer reviewed.

\section{REFERENCES}

1 American Thoracic Society, Infectious Diseases Society of America. Guidelines for the Management of Adults with Hospital-acquired, Ventilator-associated, and Healthcare-associated Pneumonia. Am J Respir Crit Care Med 2005;171:388-416.

2 World Health Organization. The World Health Report 2001. Healthy life expectancy. WHO Statistics. 2001. http://whoint/whosis/hale/halecfm

3 European Commission. The Stockholm and Barcelona targets: increasing employment of older workers and delaying the exit from the labour market. Working paper of the Commission Services Employment and Social Affairs Brussels, EMPL/A/1 2 A.D. 2002.

4 European Commission. Employment in Europe 2003. Recent trends and prospect. Employment and Social Affairs. Luxembourg: Office for Official Publications of the European Communities, 2003.

5 Berman SJ. Infections in patients with end-stage renal disease. An overview. Infect Dis Clin North Am 2001;15:709-20; vii.

6 Merrer J, Santoli F, Appere dV, et al. "Colonization pressure" and risk of acquisition of methicillin-resistant Staphylococcus aureus in a medical intensive care unit. Infect Control Hosp Epidemiol 2000:21:718-23.

7 Friedmann $R$, Raveh $D$, Zartzer $E$, et al. Prospective evaluation of colonization with extended-spectrum beta-lactamase (ESBL)-producing enterobacteriaceae among patients at hospital admission and of subsequent colonization with ESBL-producing enterobacteriaceae among patients during hospitalization. Infect Control Hosp Epidemiol 2009;30:534-42.

8 Brown RB, Cipriani D, Schulte M, et al. Community-acquired bacteremias from tunneled central intravenous lines: results from studies of a single vendor. Am J Infect Control 1994;22:149-51.

9 Valdivieso M, Gil-extremera B, Zornoza J, et al. Gram-negative bacillary pneumonia in the compromised host. Medicine (Baltimore) 1977;56:241-54.

10 Sickles EA, Young VM, Greene WH, et al. Pneumonia in acute leukemia. Ann Intern Med 1973;79:528-34.

11 Carratala J, Roson B, Fernandez-Sevilla A, et al. Bacteremic pneumonia in neutropenic patients with cancer: causes, empirical antibiotic therapy, and outcome. Arch Intern Med 1998;158:868-72.

12 Kollef MH, Shorr A, Tabak YP, et al. Epidemiology and outcomes of health-care-associated pneumonia: results from a large US database of culture-positive pneumonia. Chest 2005;128:3854-62.

13 Micek ST, Kollef KE, Reichley RM, et al. Health care-associated pneumonia and community-acquired pneumonia: a single-center experience. Antimicrob Agents Chemother 2007;51:3568-73.

14 Shindo Y, Sato S, Maruyama E, et al. Health-care-associated pneumonia among hospitalized patients in a Japanese community hospital. Chest 2009;135:633-40.

15 Schreiber MP, Chan CM, Shorr AF. Resistant pathogens in non-nosocomial pneumonia and respiratory failure: is it time to refine the definition of health-care-associated pneumonia? Chest 2010;137:1283-8.

16 Seki M, Hashiguchi K, Tanaka A, et al. Characteristics and disease severity of healthcare-associated pneumonia among patients in a hospital in Kitakyushu, Japan. J Infect Chemother 2011;17:363-9.

17 Park HK, Song JU, Um SW, et al. Clinical characteristics of health care-associated pneumonia in a Korean teaching hospital. Respir Med 2010:104:1729-35.

18 Jung JY, Park MS, Kim YS, et al. Healthcare-associated pneumonia among hospitalized patients in a Korean tertiary hospital. BMC Infect Dis 2011;11:61.

19 Garcia-Vidal C, Viasus D, Roset A, et al. Low incidence of multidrug-resistant organisms in patients with healthcare-associated pneumonia requiring hospitalization. Clin Microbiol Infect 2011;17:1659-65.

20 Carratala J, Mykietiuk A, Fernandez-Sabe N, et al. Health care-associated pneumonia requiring hospital admission: epidemiology, antibiotic therapy, and clinical outcomes. Arch Intern Med 2007;167:1393-9.

21 Polverino E, Dambrava P, Cilloniz C, et al. Nursing home-acquired pneumonia: a 10 year single-centre experience. Thorax 2010;65:354-9.

22 Chalmers JD, Taylor JK, Singanayagam A, et al. Epidemiology, antibiotic therapy, and clinical outcomes in health care-associated pneumonia: a UK cohort study. Clin Infect Dis 2011:53:107-13.

23 Ewig S, Welte T, Torres A. Is healthcare-associated pneumonia a distinct entity needing specific therapy? Curr Opin Infect Dis 2012;25:166-75.

24 Shorr AF, Zilberberg MD, Micek ST, et al. Prediction of infection due to antibiotic-resistant bacteria by select risk factors for health care-associated pneumonia. Arch Intern Med 2008;168:2205-10.

25 Fine MJ, Auble TE, Yealy DM, et al. A prediction rule to identify low-risk patients with community-acquired pneumonia. N Engl J Med 1997;336:243-50.

26 Lim WS, van der Eerden MM, Laing R, et al. Defining community acquired pneumonia severity on presentation to hospital: an international derivation and validation study. Thorax 2003;58:377-82.

27 Menendez R, Torres A, Aspa J, et al. [Community acquired pneumonia. New guidelines of the Spanish Society of Chest Diseases and Thoracic Surgery (SEPAR)] Arch Bronconeumol 2010;46:543-58.

28 Mandell LA, Wunderink RG, Anzueto A, et al. Infectious Diseases Society of America/American Thoracic Society consensus guidelines on the management 


\section{Respiratory infection}

of community-acquired pneumonia in adults. Clin Infect Dis 2007:44(Suppl 2): S27-72.

29 Murray TJ, Washington JA. Microscopic and bacteriologic analysis of expectorated sputum. Mayo Clin Proc 1975;50:339-44.

30 Rano A, Agusti C, Jimenez $\mathrm{P}$, et al. Pulmonary infiltrates in non-HIV immunocompromised patients: a diagnostic approach using non-invasive and bronchoscopic procedures. Thorax 2001;56:379-87.

31 Fischer JE, Bachmann LM, Jaeschke R. A readers' guide to the interpretation of diagnostic test properties: clinical example of sepsis. Intensive Care Med 2003:29:1043-51.

32 Laheij RJ, Sturkenboom MC, Hassing RJ, et al. Risk of community-acquired pneumonia and use of gastric acid-suppressive drugs. JAMA 2004:292:1955-60.

33 Sarkar M, Hennessy S, Yang YX. Proton-pump inhibitor use and the risk for community-acquired pneumonia. Ann Intern Med 2008;149:391-8.
34 Gulmez SE, Holm A, Frederiksen $\mathrm{H}$, et al. Use of proton pump inhibitors and the risk of community-acquired pneumonia: a population-based case-control study. Arch Intern Med 2007;167:950-5.

35 Marrie TJ, Wu L. Factors influencing in-hospital mortality in community-acquired pneumonia: a prospective study of patients not initially admitted to the ICU. Chest 2005; 127:1260-70.

36 Sligl WI, Eurich DT, Marrie TJ, et al. Only severely limited, premorbid functional status is associated with short- and long-term mortality in patients with pneumonia who are critically ill: a prospective observational study. Chest 2011;139:88-94.

37 Pavia AT. What is the role of respiratory viruses in community-acquired pneumonia? What is the best therapy for influenza and other viral causes of community-acquired pneumonia? Infect Dis Clin North Am 2013;27:157-75.

38 Ewig S, Klapdor B, Pletz MW, et al. Nursing-home-acquired pneumonia in Germany: an 8-year prospective multicentre study. Thorax 2012;67:132-8. 


\section{Correction}

Polverino E, Torres A, Menendez R, et al. Microbial aetiology of healthcare associated pneumonia in Spain: a prospective, multicentre, case-control study. Thorax 2013;68:1007-14. doi:10.1136/thoraxjnl-2013-203828.

The date reported under the row 'Pleural effusion' in table 3 of this published paper is incorrect. The correct data for that row is below:

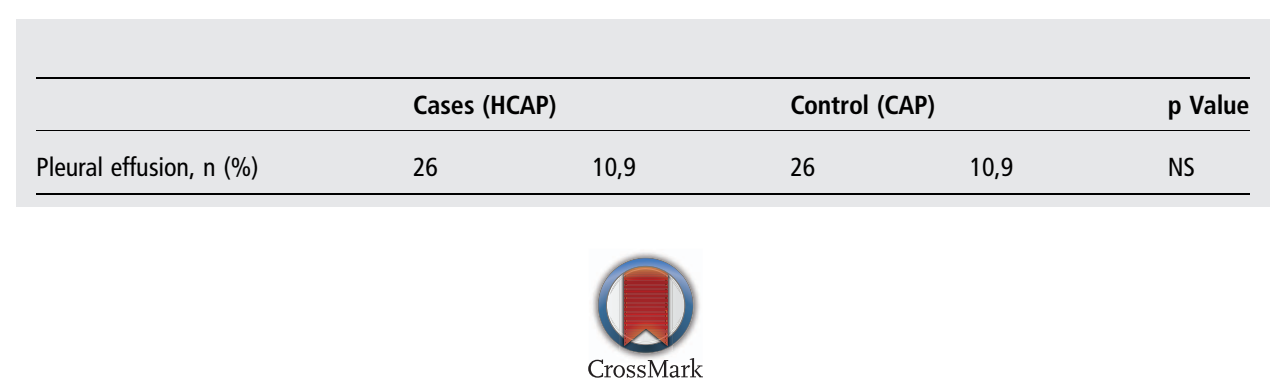

Thorax 2015;70:708. doi:thoraxjnl-2013-203828corr1 